International Journal of Engineering \& Technology, $7(4.14)(2018) 116-120$
International Journal of Engineering \& Technology
SPC
Website: www.sciencepubco.com/index.php/IJET
Research paper

\title{
Comparative Study on Anti-Acetylcholinesterase and Anti-Inflammatory Activities of Date and Apple Vinegars Fortified with Centella Asiatica
}

\author{
M I Adenan ${ }^{1,2 *}$, N A Jusril, ${ }^{1,2}, \mathrm{~K}$ A Radzun ${ }^{1}, \mathrm{Z}$ Z Hafiz ${ }^{1,2}$ \\ ${ }^{1}$ Faculty of Applied Sciences, Universiti Teknologi MARA, UiTM Shah Alam, 40450 Shah Alam, Selangor, Malaysia \\ ${ }^{2}$ Atta-ur-Rahman Institute for Natural Product Discovery, Aras 9, Bangunan FF3, UiTM Puncak Alam, 42300 \\ Puncak Alam, Selangor, Malaysia \\ *Corresponding author E-mail: ilhamipharm@gmail.com
}

\begin{abstract}
Neuroinflammation and deficiency of cholinergic are major factors of neurodegenerative damage correlated to cognitive impairment in Alzheimer's disease (AD). We investigated the anti-inflammatory and anti-acetylcholinesterase activities of apple and date vinegars added with Centella asiatica in SH-SY5Y neuroblastoma cells. The neuroprotective effect of apple or date vinegar added with various percentage of $C$. asiatica $(0,0.5,2,5) \% \mathrm{w} / \mathrm{v}$ was determined in vitro. The methanolic extract of apple vinegar added with $2 \%$. asiatica (AV-2\% CA) and date vinegar added with $2 \%$ C. asiatica (DV-2\% CA) -extracts showed potent neuroprotective effect. Both extracts were subjected to liquid-liquid partitioning yielded aqueous $\left(\mathrm{H}_{2} \mathrm{O}\right.$ : AV/DV-2\%_CA) and ethyl acetate (EA: AV/DV-2\% CA) extracts. Anti-inflammatory response against nitric oxide (NO) of all extracts was measured in LPS-induced SH-SY5Y neuroblastoma cells and percentage inhibition of acetylcholinesterase (AChE) was measured using commercially available test kits. It was found that EA: DV-2\% CA showed potent ameliorating effect against LPS-induced inflammation $\left(\mathrm{I}_{50}: 563.5 \pm 0.13 \mu \mathrm{g} / \mathrm{mL}\right)$ and was also responsible in the AChE inhibition activity $\left(\mathrm{IC}_{50}: 9.087 \pm 0.02\right)$. Thus, this extract is suggested to have dual properties of anti-inflammatory and antiacetylcholinesterase inhibition activity that could be beneficial in the treatment and prevention of neurodegenerative disease.
\end{abstract}

Keywords: Apple vinegar, date vinegar, Centella asiatica, acetylcholinesterase inhibition, anti-inflammation

\section{Introduction}

Alzheimer's disease (AD) is a neurodegenerative disorder of the central nervous system (CNS) that is associated with low levels of acetylcholine neurotransmitter and loss of cholinergic cells in the brain [1]. There are several pathological features identified in the central nervous system (CNS) of AD patients such as neurofibrillary tangles, inflammatory processes, amyloid plaques and disruption of neurotransmitters [2]. Centella asiatica (CA) or locally known as pegaga is one the medicinal plants used for wound healing, skin problems treatment, and brain and nerves cells revitalization [3].

The therapeutic action of this medicinal plant is mainly attributed due to the presence of bioactive compounds such as asiaticoside, madecassoside, madecassic acid and asiatic acid [4]. Vinegar has long been part of human diet as food preservative and simple remedies for human and animal. There are many types of raw materials used in the production of vinegar such as grapes, berry and others [5]. It has been reported that apple vinegar contains polyphenolic, vitamin, organic acid, flavonoids and mineral elements which contribute to variety of pharmacological functions [6]. It is also reported that the beneficial effects of date vinegar may in part be correlated to its polyphenolic compounds [7].

Neuroinflammation has been identified related to $\mathrm{AD}$ and may contribute to neuronal injury and hyperphosphorylation [8]. Inflammation and chronic neuro injury could weakened the brain pathways which typically reduce and damage the synaptic transmission and neural network [9].

There is limited research being done so far on the combination of vinegar and herbs toward the anti-acetylcholinesterase and antiinflammatory activities. However, vinegar added with 3\% of Akebia quinata young leaves extract was demonstrated to show excellent biological activities [10]. It is due to the presence of phenolic compounds in both combinations of extracts.

In the present study, the SH-SY5Y neuroblastoma cell line was selected as a model for the in vitro study of proliferation rate of neuronal cell induced by bacterial Lipopolysaccharide (LPS). The SH-SY5Y cell line is an ideal for in vitro model for neurotoxicity, neuroprotective, and other factors related to the neurodegenerative process [11].

Therefore, the aim of this study was to delineate the effects of $C$. asiatica extract supplementation to apple or date vinegars on neuroprotective role of LPS-induce inflammation in SH-SY5Y neuroblastoma cell.

\section{Materials and Methods}

\subsection{Preparation of extracts}

Apple and date vinegars were purchased from Mahnaz Food, Kuala Lumpur, Malaysia. CA extract was obtained from the Atta-urRahman Institute of Natural Product Discovery (AuRIns), 
Universiti Teknologi MARA (UiTM) Selangor (Puncak Alam Campus), Selangor Malaysia. CA extract at four level percentages $(0,0.5,2$, and $5 \% \mathrm{w} / \mathrm{v})$ were mixed with $200 \mathrm{ml}$ of apple vinegar (AV) liquid for one week. The mixture was filtered to obtain an apple vinegar- $C$. asiatica liquid. Sodium carbonate was then added to the liquid with a 1:10 ratio. The liquid mixture was boiled until it became $1 / 10^{\text {th }}$ of its size and allowed to solidify to produce apple vinegar- $C$. asiatica (AV-CA) powder. This method was repeated for date vinegar - $C$. asiatica (DV-CA).

Methanolic extracts of AV-CA or DV-CA were obtained by extracting $10 \mathrm{~g}$ of AV-CA or DV-CA with methanol in a rotary shaker for $12 \mathrm{~h}$ and filtered by using a Whatman No.4 filter paper. The methanolic extracts obtained were concentrated in a rotary evaporator at $40^{\circ} \mathrm{C}$ and kept at $4{ }^{\circ} \mathrm{C}$ for further analysis.

Ethyl acetate and water extracts of AV-CA or DV-CA were prepared by partitioning $10 \mathrm{~g}$ of methanol extracts of AV-CA or DVCA with $50 \mathrm{~mL}$ of distilled water and $30 \mathrm{~mL}$ of ethyl acetate. The ethyl acetate filtrate was concentrated using a rotary evaporator at $60^{\circ} \mathrm{C}$ to obtain ethyl acetate extract of AV-CA (EA: AV-CA). While, water layer was freeze dried for three days to obtain water extract of AV-CA $\left(\mathrm{H}_{2} \mathrm{O}: \mathrm{AV}-\mathrm{CA}\right)$ and kept at $4^{\circ} \mathrm{C}$ for further analysis. The experiment was repeated for methanolic extract of DVCA to obtain ethyl acetate extract of DV-CA (EA: DV-CA) and water extract of DV-CA $\left(\mathrm{H}_{2} \mathrm{O}\right.$ : DV-CA).

\subsection{Cell Culture}

SH-SY5Y neuroblastoma cells were purchased from ATCC (ATCC CRL-2266). SH-SY5Y cells was cultured in a 1:1 mixture of Dulbecco's Modified Eagle Medium (DMEM) and Nutrient Mixture Ham's F12 Medium and supplemented with 10\% (v/v) heat-inactivated fetal bovine serum (FBS) with $1 \% \quad(\mathrm{v} / \mathrm{v})$ PEN/STREP and filtered through a $0.22 \mathrm{uM}$ pore filter apparatus. The cells were grown in $75 \mathrm{~cm}^{3}$ flask with $10 \mathrm{ml}$ medium at seeding density of 1-1.5 x $10^{4}$ cells.

\subsection{3-(4,5-dimethylthiazol-2-yl)-2.5-diphenyltetrazolium bromide (MTT) assay}

An MTT assay was performed as described previously [12]. Cell viability was measured by MTT colorimetric assay (SigmaAldrich, St. Louis, MO, USA). After incubation, $20 \mu \mathrm{L}$ of MTT $(5 \mathrm{mg} / \mathrm{mL})$ was added to each well and the cells were cultured for another four hours. The medium was next removed and $100 \mu \mathrm{L}$ of DMSO was added to each well to dissolve the formazan. The absorbance was evaluated using a microplate reader (BMG, LABTECH) at $540 \mathrm{~nm}$

\subsection{Neurotoxicity assay}

SH-SY5Y neuroblastoma cells of more than $80 \%$ confluence were used for the assay [13]. Cells were seeded in 96-microwell plate with cell density $\left(\begin{array}{lll}1 & \times & 4\end{array}\right)^{4}$ cells per well and incubated overnight to allow sufficient cells attachment. The medium was discarded and the working solution $(100 \mu \mathrm{L})$, which was diluted with the medium to yield the final concentrations of the test solution ranging from $7.813-1000 \mu \mathrm{g} / \mathrm{mL}$ were added into the well and incubated for $24 \mathrm{~h}$. As a blank, cells were cultured in just $100 \mu \mathrm{L}$ of medium. All treatments were done in triplicate. Viability of the cells was determined using MTT assay according to manufacturer's instruction.

\subsection{Neuroprotective assay}

The neuroprotective effects of methanolic AV-CA or DV-CA extracts were investigated on LPS-induced inflammation in $\mathrm{SH}$ -
SY5Y cells. Cells were treated with a range of concentration of extracts from 7.813-1000 $\mu \mathrm{g} / \mathrm{mL}$ and for $24 \mathrm{~h}$. Dexamethasone at $2.806 \mu \mathrm{M}$ was used as a positive control. After $24 \mathrm{hr}$ of pretreatment, cells were exposed to optimal concentration of LPS $(4.94 \mu \mathrm{g} / \mathrm{mL})$ as pre-determined in this study, for $24 \mathrm{~h}$. The cell viability was measured by using MTT assay[14].

\subsection{Preparation of cell lysates}

SH-SY5Y cells from each well were harvested after drug treatment. The well-plates containing culture medium were washed with cold PBS, and then pallets cells were produced by centrifugation at $10,000 \mathrm{rpm}$ for $10 \mathrm{~min}$. The supernatant was removed and resuspended with ice-cold RIPA lysis and extraction buffer (Millipore Corporation, Billerica, MA, USA) and incubated on ice for 30 minutes.

\subsection{Nitrite assay}

The nitric oxide (NO) production was determined through the indication level of nitrite in cells media. The SH-SY5Y cells were seeded in 6 well-plates $\left(1 \times 10^{6}\right.$ cells/well $)$ with $2 \mathrm{~mL}$ of cell culture media and incubated for $24 \mathrm{~h}$. Then, the old media were discarded and replaced with new media to maintain the cells. Various concentration of ethyl acetate and water extracts (AV-CA and DV-CA) from $7.8125-1000 \mu \mathrm{g} / \mathrm{mL}$ and the positive control of dexamethasone were pre-treated with the SH-SY5Y neuroblastoma cells. Cells were induced with LPS for all samples for another $24 \mathrm{~h}$. Then, cells were subjected to lysate preparation by using RIPA lysis and extraction buffer according to the manufacturer's protocol. Then, $100 \mu \mathrm{L}$ of collected supernatant from lysate were transferred into 96-well plate in triplicates. The plates were then incubated with Griess reagent followed by Nitrite/nitrile colorimetric assay according to the kit's protocol (Cayman Chemical) and incubated in room temperature for $10 \mathrm{~min}$ in dark condition. The absorbance was determined by using microplate reader at $540 \mathrm{~nm}$ wavelength.

\subsection{Acetylcholinesterase assay}

Cells were plated in 6-well plate at a density $1 \times 10^{6}$ cells $/ \mathrm{mL}$. After incubation for two days, $10 \mu \mathrm{M}$ all-trans-RA was added to the medium and incubated for another two days. After 2 days of neurite outgrowth, cells were treated with various concentrations (0.781 to $1000 \mu \mathrm{g} / \mathrm{mL})$ of EA: AV-CA/EA: DV-CA and $\mathrm{H}_{2} \mathrm{O}: \mathrm{AV}$ $\mathrm{CA} / \mathrm{H}_{2} \mathrm{O}$ : DV-CA. The morphological changes in the cells were observed under phase contrast microscope. Then, cells were subjected to lysate preparation by using RIPA buffer according to the manufacturer's protocol. Cholinergic dysfunction was determined by measuring acetylcholinesterase activity level using QuantiChrom $^{\mathrm{TM}}$ Acetylcholinesterase Assay Kit (BioAssay System, CA, USA). Eserine was used as a positive control.

\subsection{Statistically analysis}

Each experiment was carried out at least in triplicate and comparative analysis of the triplicate data was conducted. Data are represented as mean \pm standard error of the mean (SEM) of 3 replicates. The statistical analyses amongst different group was performed by two-way analysis of variance (ANOVA) followed by Post Hoc Tukey's Comparison Test using GraphPad PRISM Version 6.0, whereby positive significant results were indicated as asterisks $(* P<0.05, * * P<0.01, * * * P, 0.001, \mathrm{a}=* * *)$

\section{Results and Discussion}


The neurotoxicity of methanolic extracts of AV-CA or DV-CA at the concentration of $250 \mu \mathrm{g} / \mathrm{mL}$ on cell viability of SH-SY5Y cells were examined using MTT assay. Based on the result, the mean cell viability was increased after treatment with methanolic AV$\mathrm{CA}$ and DV-CA extracts after $24 \mathrm{~h}$ as compared to untreated control. However, the mean cell viability was not significantly reduced after pre-treatment with CA alone ( $p<0.001$; Fig 1). MTT assay is an accurate and defensive test, with color development which strongly correlating with cell numbers. It is widely used in a cytotoxicity test [15]. It was observed that methanolic extracts of AV-CA and DV-CA showed no toxicity effects on SH-SY5Y cells as the cell viability was increased as compared to untreated control (Fig 1).

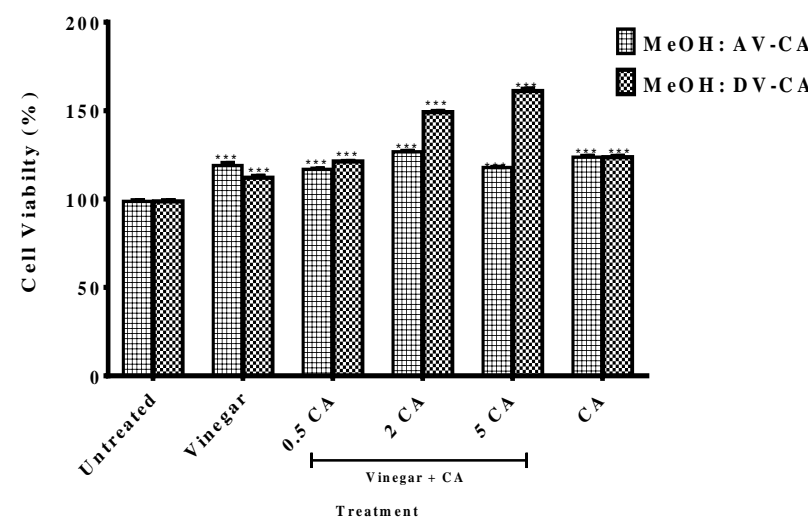

Fig 1: Effect of methanolic AV-CA and DV-CA extracts [different percentage of CA $(0,0.5,2$, and $5 \% \mathrm{w} / \mathrm{v})]$ on cell viability of SH-SY5Y cells for $24 \mathrm{~h}$, cell viability was assessed with MTT assay. Data are expressed as mean $\pm \mathrm{SD}(\mathrm{n}=3)$ of percentage to untreated control. $* * * \mathrm{P}=0.001 \mathrm{Com}-$ pared to untreated control.

SH-SY5Y cells were pre-treated with methanolic extracts of AVCA and DV-CA at a concentration of $250 \mu \mathrm{g} / \mathrm{ml}$ for $24 \mathrm{~h}$, and cells were then induced with LPS $(42.19 \mu \mathrm{g} / \mathrm{ml})$ for another $24 \mathrm{~h}$. The cell viability was determined by metabolic reduction as determined by metabolic reduction of a tetrazolium salt to a formazan dye (MTT assay). The LPS-induced cell death $(50 \%$ of viable cells) was significantly restored by pre-treatment with AV-CA and DV-CA added with 0.5, 2 and 5\% CA. Pre-treatment with methanolic AV-CA and DV-CA extracts could protect the cells from the LPS-induced cell death. It has been reported that asiatic acid found in CA has preventive effect on colon tumorigenesis and good healing activities [3]. It was observed that pre-treatment with methanolic extracts of AV-CA and DV-CA added with $2 \%$ CA showed highest neuroprotective effect on LPS-induced SH-SY5Y cells (Fig 2). Therefore, AV-CA and DV-CA added with 2\% CA were subjected to liquid-liquid extraction using ethyl acetate with water.

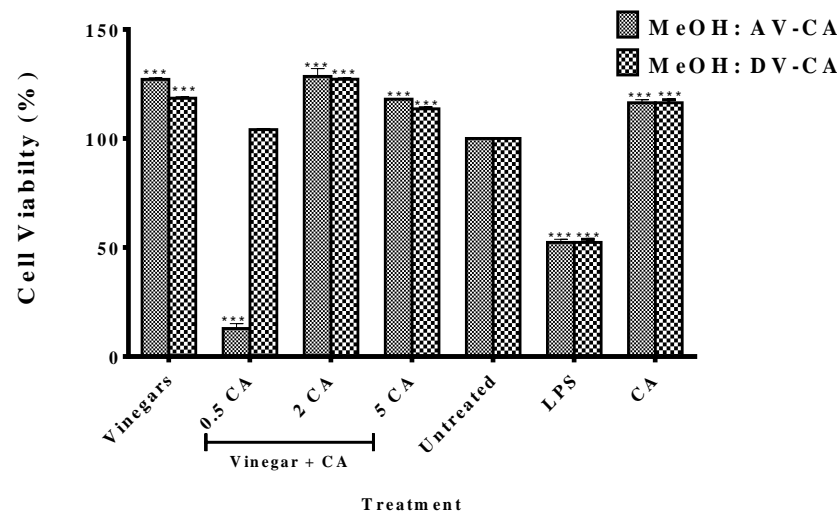

Fig 2: Neuroprotective effects of methanolic AV-CA and DV-CA extracts [different percentage of CA $(0,0.5,2$, and $5 \%$ ] on cell viability of LPSinduced SH-SY5Y cells for $24 \mathrm{~h}$, cell viability was measured by using
MTT assay. Significant differences from untreated are indicated by representing $\mathrm{p}^{* * *}$ value $<0.001$

Nitric oxide (NO), which is synthesized by enzyme nitric oxide synthase (NOS), also plays important role in many physiological conditions. It has been reported, LPS inducement was found to stimulate NO production and iNOS expression [16]. The effects of EA: AV-CA/DV-CA and $\mathrm{H}_{2} \mathrm{O}$ : AV-CA/DV-CA with [2\% CA] extracts on NO production in LPS-induced SH-SY5Y cells were tested using NO assay. Fig 3 shows the nitrite concentration in all extracts using NO assay. Cells were pre-treated with extracts for $24 \mathrm{~h}$ at various concentrations before stimulated with LPS for additional $24 \mathrm{~h}$. Results showed a significant concentrationdependent inhibition of nitrite production in the presence of EA: AV-CA/DV-CA and $\mathrm{H}_{2} \mathrm{O}$ : AV-CA/DV-CA [with $2 \% \mathrm{CA}$ ]. It was found that ethyl acetate extract of AV-CA added with 2\% CA [EA DV-CA ( $2 \%$ CA)] extract showed better NO production inhibition and induced more attenuation effect on nitrite production with an $\mathrm{IC}_{50}$ value of $563.5 \pm 0.13 \mu \mathrm{g} / \mathrm{mL}$ as compared to other extracts. Half maximal inhibitory concentration $\left(\mathrm{IC}_{50}\right)$ of extracts along with positive control are summarized in Table 3.1.

Table 3.1: In vitro anti-inflammatory and anti-acetylcholinesterase activities of AV-CA and DV-CA extracts

\begin{tabular}{|c|c|c|}
\hline \multirow{2}{*}{ Extracts } & \multicolumn{2}{|c|}{$\mathrm{IC}_{50}$ Values $(\mu \mathrm{g} / \mathrm{mL})$} \\
\cline { 2 - 3 } & Nitrite assay & Acetylcholinesterase assay \\
\hline EA: AV-CA $(2 \%)$ & $817.0 \pm 0.09$ & $815.6 \pm 0.84$ \\
\hline $\mathrm{H}_{2} \mathrm{O}:$ AV-CA $(2 \%)$ & $803.1 \pm 0.06$ & $49.3 \pm 1.61$ \\
\hline EA: DV-CA $(2 \%)$ & $563.5 \pm 0.13$ & $9.087 \pm 0.02$ \\
\hline $\mathrm{H}_{2} \mathrm{O}:$ DV-CA $(2 \%)$ & $>1000$ & $11.5 \pm 0.03$ \\
\hline Dexamethasone & $2.113 \pm 0.06$ & $\mathrm{ND}$ \\
\hline Eserine & $\mathrm{ND}$ & $6.62 \pm 0.04$ \\
\hline
\end{tabular}

ND: Not Determined

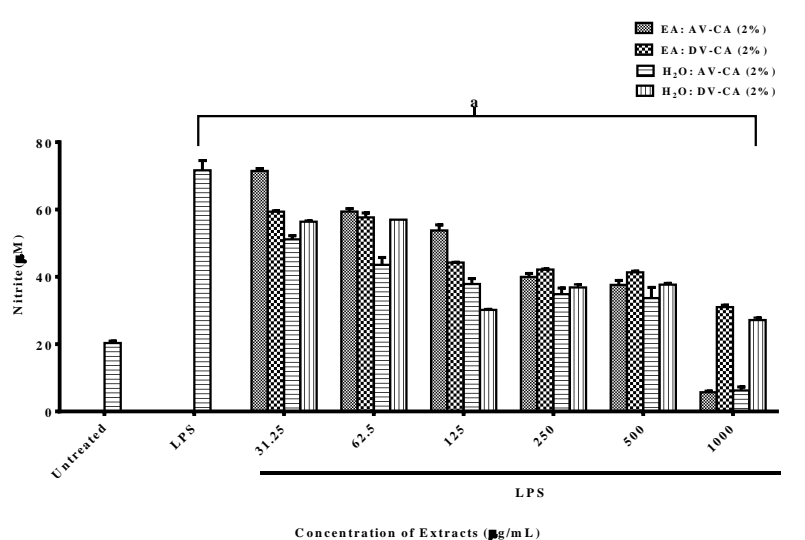

Fig 3: Effect of EA: AV-CA/DV-CA and $\mathrm{H}_{2} \mathrm{O}$ : AV-CA/DV-CA at [2\% $\mathrm{CA}]$ extracts on LPS-Induced nitrite production in SH-SY5Y neuroblastoma cells. After treated by LPS $(42.19 \mathrm{mg} / \mathrm{mL})$ with or without extracts $(7.8125-1000 \mathrm{mg} / \mathrm{mL})$, the amount of nitrite in the culture supernatants were measured by the griess reaction assay. Each column represents the mean \pm SEM. The experimental results are statistically analyzed in comparisons with untreated control; Where ${ }^{\mathrm{a}} \mathrm{p}=0.001$.

Acetycholinesterase (AChE) is one of the most required enzymes in the group of serine hydrolyses which responsible in cognition and memory, where it will catalyze the breakdown of neurotransmitter acetylcholine. This enzyme is also well known in neural transmission. The deficiency of cholinergic is the main cause of $\mathrm{AD}$ [17]. EA: AV-CA/DV-CA and $\mathrm{H}_{2} \mathrm{O}$ : AV-CA/DV-CA [2\% CA] extracts were tested against AChE enzyme inhibition activity and the percentage inhibition was evaluated. Inhibitory concentration data of extracts revealed that the strongest $\mathrm{AChE}$ inhibition activity was exhibited by EA: DV-CA $(2 \%$ CA $)$ extract with $\mathrm{IC}_{50}=$ $9.087 \mu \mathrm{g} / \mathrm{ml}$ which was better than $\mathrm{H}_{2} \mathrm{O}$ : DV-CA $(2 \%)$ extract with $\mathrm{IC}_{50}=11.55 \mu \mathrm{g} / \mathrm{ml}$ (Table 3.1). Meanwhile, inhibitory concentration data of $\mathrm{H}_{2} \mathrm{O}$ : AV-CA $(2 \%)$ showed stronger inhibition with $\mathrm{IC}_{50}=49.30 \mu \mathrm{g} / \mathrm{ml}$ as compared to EA: AV-CA (2\%) with $\mathrm{IC}_{50}=815.6 \mu \mathrm{g} / \mathrm{ml}$. All extracts were compared with standard 
drug of Eserine with $\mathrm{IC}_{50}=5.905 \mu \mathrm{g} / \mathrm{ml}$. Results from AChE enzyme inhibition activity clearly indicated that EA: DV-CA $(2 \%)$ showed a potent inhibition followed by $\mathrm{H}_{2} \mathrm{O}$ : DV-CA (2\%) with moderate activity when compared to control (Fig 4).

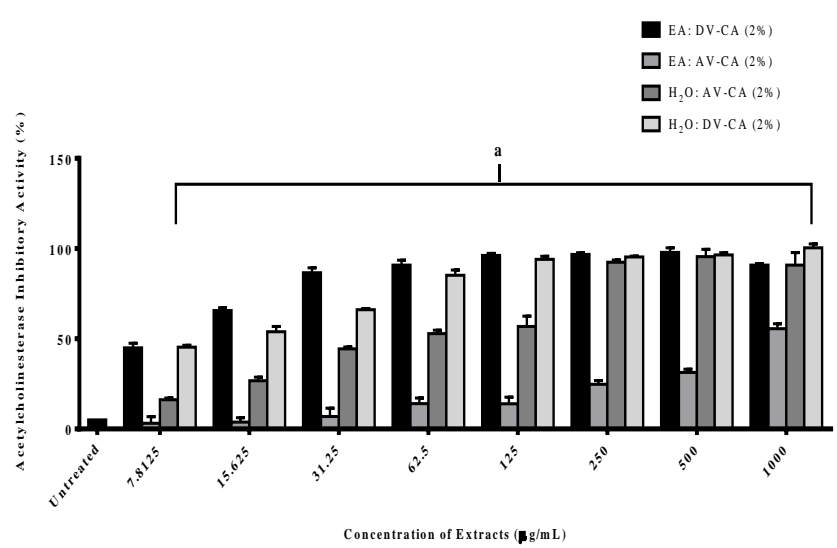

Fig 4: Effects of EA: AV-CA/DV-CA and $\mathrm{H}_{2} \mathrm{O}$ : AV-CA/DV-CA at [2\% $\mathrm{CA}$ ] on AChE activity in SH-SY5Y neuroblastoma cells. Each column represents the Mean \pm SEM. The experimental results are statistically analyzed in comparisons with untreated control ; Where ${ }^{\mathrm{a}} p=0.001$

The inhibition of enzyme AChE by the ethylacetate extract of date vinegar added with $2 \%$ CA [EA: DV-CA (2\% CA)] might be able to increase the level of neurotransmitter acetylcholine and thus improve synaptic transmission in $\mathrm{AD}$ brain. It is well understood that the most remarkable changes in $\mathrm{AD}$ patients is reduction of acetylcholine (ACh) levels in the cortex and hippocampus of the brain. Therefore, inhibition of AChE, in which the enzyme responsible for hydrolysis of ACh at the cholinergic synapse interaction can be the appropriate target in the treatment of $\mathrm{AD}$ [18].

EA: DV-CA (2\%) showed potent ameliorating effect against LPSinduced inflammation and was responsible in the AChE inhibition activity. Thus, this extract has dual properties of antiinflammatory and anti-acetylcholinesterase inhibition. Therefore, neuroprotective effect of EA: DV-CA (2\%) extract could be beneficial in the treatment and prevention of neurodegenerative disease. The presence of polyphenols and nutrients such as amino acids, peptides, vitamins and minerals could enhance the health benefits of apple and date vinegars fortified with Centella asiatica.

\section{Conclusion}

The ethyl acetate extract of date vinegar added with 2\% CA [EA: DV-CA $(2 \%)$ ] showed potent ameliorating effect against LPSinduced inflammation and was responsible in the AChE inhibition activity. Thus, this extract has dual properties of antiinflammatory and anti-acetylcholinesterase inhibition. Therefore, the presence of semi polar and polar compounds with neuroprotective activity in the EA: DV-CA $(2 \%)$ extract could be beneficial in the treatment and prevention of neurodegenerative disease.

\section{Acknowledgement}

The authors gratefully acknowledge the Ministry of Agriculture, Malaysia [MOA Grant no. 100-RMI-/MOA 16/6/2(5/2015)], AttaUr-Rahman Institute for Natural Product Discovery (AuRins) and University Teknologi MARA (UiTM) for funding and providing facilities throughout this study.

\section{References}

[1] J. Park, S. Shin, K. C. Park, E. Jeong, and J. H. Park, "Synthesis and in vitro Assay of New Triazole Linked Decursinol Derivatives Showing Inhibitory Activity against Cholinesterase for Alzheimer's Disease Therapeutics," J. Korean Chem. Soc., vol. 60, no. 2, pp. 125-130, 2016

[2] A. H. Nour et al., "In vitro Anti-Acetylcholinesterase and Antioxidant Activity of Selected Malaysian Plants," Asian J. Pharm. Clin. Res., vol. 7, no. 3, pp. 93-97, 2014.

[3] J. J. J. Zhang et al., "Wound Healing Activities of Different Extracts of Centella asiatica in Incision and Burn Wound Models: An Experimental Animal Study.," BMC Complement. Altern. Med., vol. 3, no. 1, pp. 8-14, 2013.

[4] A. Prasad, M. Singh, N. P. Yadav, A. K. Mathur, and A. Mathur, "Molecular, Chemical and Biological Stability of Plants Derived From Artificial Seeds of Centella asiatica (L.) Urban-An Industrially Important Medicinal Herb," Ind. Crops Prod., vol. 60, pp. 205-211, 2014

[5] A. Mas, M. J. Torija, G. Carmne, A-Parrilla, and T. M. Ana, "Acetic Acid Bacteria and the Production and Quality of Wine Vinegar, Acetic Acid Bacteria and the Production and Quality of Wine Vinegar," Sci. World J., vol. 2014, pp. 1-6, 2014.

[6] S. Pourmozaffar, A. Hajimoradloo, and H. K. Miandare, "Dietary effect of apple cider vinegar and propionic acid on immune related transcriptional responses and growth performance in white shrimp, Litopenaeus vannamei," Fish Shellfish Immunol., vol. 60, pp. 65-71, 2017.

[7] M. H. Matloob, A. Alaa, and A. B. D. A. Balakit, "Phenolic Content of Various Date Palms Fruits and Vinegars From Iraq," vol 14, no. 4, pp. 1893-1906, 2016

[8] J. Popp et al., "Markers of Neuroinflammation Associated with Alzheimer's Disease Pathology in Older Adults," Brain. Behav. Immun., vol. 62, no. 62, pp. 203-211, 2017.

[9] M. Rahimifard et al., "Targeting the TLR4 Signaling Pathway by Polyphenols: A Novel Therapeutic Strategy for Neuroinflammation," Ageing Res. Rev., vol. 36, no. 6, pp. 11-19, 2017.

[10] W. Y. Kwon et al., "Quality characteristics and biological activities of vinegars added with young leaves of Akebia quinata," J. Korean Soc. Food Sci. Nutr., vol. 43, no. 7, pp. 989-998, 2014.

[11] H. J. Heusinkveld and R. H. S. Westerink, "Comparison of different in vitro cell models for the assessment of pesticide-induced dopaminergic neurotoxicity," Toxicol. Vitr., vol. 45, no. July, pp. $81-88,2017$.

[12] K. Khwanraj, C. Phruksaniyom, S. Madlah, and P. Dharmasaroja, "Differential Expression of Tyrosine Hydroxylase Protein and Apoptosis-Related Genes in Differentiated and Undifferentiated SH-SY5Y Neuroblastoma Cells Treated with MPP+," Neurol. Res. Int., vol. 2015, 2015.

[13] G. Sala et al., "Rotenone upregulates alpha-synuclein and myocyte enhancer factor 2D independently from lysosomal degradation inhibition," Biomed Res. Int., vol. 2013, no. March 2014, 2013.

[14] M. Ramkumar et al., "Neuroprotective effect of Demethoxycurcumin, a natural derivative of Curcumin on rotenone induced neurotoxicity in SH-SY 5Y Neuroblastoma cells," $B M C$ Complement. Altern. Med., vol. 17, no. 1, pp. 1-11, 2017.

[15] Y. Gao et al., "Anti-Inflammatory Effects of Sophocarpine in LPSInduced RAW 264.7 Cells Via NF-kB and MAPKs Signaling Pathways," Toxicol. Vitr., vol. 26, no. 1, pp. 1-6, 2012.

[16] S.-K. Heo, H.-J. Yun, E.-K. Noh, W.-H. Park, and S.-D. Park, "LPS induces inflammatory responses in human aortic vascular smooth muscle cells via Toll-like receptor 4 expression and nitric oxide production," Immunol. Lett., vol. 120, no. 1, pp. 57-64, 2008.

[17] S.-H. Lu et al., "The discovery of potential acetylcholinesterase inhibitors: a combination of pharmacophore modeling, virtual screening, and molecular docking studies.," J. Biomed. Sci., vol. 18 , no. 1 , p. $8,2011$.

[18] H. Saleem et al., "In vitro acetylcholinesterase and butyrylcholinesterase inhibitory potentials of Jatropha gossypifolia plant extracts," Acta Pol. Pharm. - Drug Res., vol. 73, no. 2, pp. 419-423, 2016. 